\title{
Non Linear Thermal Decaying Functions
}

\author{
Espinosa Fuentes EA, PhD. ${ }^{1}$, Valdez Cervantes LC, MSc. ${ }^{1}$ and Colpas Castillo F, MSc. ${ }^{2}$ \\ ${ }^{1}$ TECNAR, Colombia, eduardo.espinosa@tecnar.edu.co, libis.valdez@tecnar.edu.co \\ ${ }^{2}$ University of Cartagena, Colombia, fcolpas2@unicartagena.edu.co
}

\begin{abstract}
The present manuscript deals with a deep mathematical analysis of the functions that should be used in the Clausius Clapeyron thermodynamic modelling, which is usually modelled linearly, this research emphasizes that the thermal models are nonlinear even taking into account the heat capacities as a constant. Specifically, the mathematical analysis of the thermal decaying models proposes what equations should be used depending on the type of enthalpy function. Consequently, the enthalpy functions depend on the model of the heat capacity proposed by Albert Einstein. The magnitude of the errors that would be committing by using linear regressions on experimental data were also estimated; as is the case for the vast majority of publications in this area. The study also shows how the models behave in wide temperature range; and how they are becoming apparent linear as the temperature range decreases.

Keywords-Clausius Clapeyron, heat capacities.
\end{abstract}

Digital Object Identifier (DOI): http://dx.doi.org/10.18687/LACCEI2015.1.1.278

ISBN: 13 978-0-9822896-8-6

ISSN: 2414-6668

$1^{\text {th }}$ LACCEI Annual International Conference: “Engineering Education Facing the Grand Challenges, What Are We Doing?” July 29-31, 2015, Santo Domingo, Dominican Republic ISBN: 13 978-0-9822896-8-6

ISSN: $2414-6668$

DOI: http://dx.doi.org/10.18687/LACCEI2015.1.1.278 


\title{
NON LINEAR THERMAL DECAYING FUNCTIONS
}

\author{
Espinosa Fuentes EA, Ph.D ${ }^{1}$, Valdez Cervantes LC, M.Sc ${ }^{1}$ and Colpas Castillo F, M.Sc ${ }^{2}$ \\ ${ }^{1}$ TECNAR, Colombia, eduardo.espinosa@tecnar.edu.co, libis.valdez@tecnar.edu.co \\ ${ }^{2}$ University of Cartagena, Colombia, fcolpas2@unicartagena.edu.co
}

\begin{abstract}
The present manuscript deals with a deep mathematical analysis of the functions that should be used in the Clausius Clapeyron thermodynamic modelling, which is usually modelled linearly, this research emphasizes that the thermal models are nonlinear even taking into account the heat capacities as a constant. Specifically, the mathematical analysis of the thermal decaying models proposes what equations should be used depending on the type of enthalpy function. Consequently, the enthalpy functions depend on the model of the heat capacity proposed by Albert Einstein. The magnitude of the errors that would be committing by using linear regressions on experimental data were also estimated; as is the case for the vast majority of publications in this area. The study also shows how the models behave in wide temperature range; and how they are becoming apparent linear as the temperature range decreases.

Keywords-- Clausius Clapeyron, heat capacities.
\end{abstract}

\section{I.INTRODUCTION}

Thermodynamic properties are important to take them into account in the development of technologies for chemical assays and energy process efficiency studies [1-4], as well as in the transport and handling of bulk quantities. The analysis methods based on thermogravimetry, Langmuir and ClausiusClapeyron expression have demonstrated to be accurate for many materials over a narrow temperature range; and they have been used extensively in the determination of many thermodynamic properties of materials with ideal behavior, in the pharmaceutical [5-9]; the applications also include the examination of antioxidants [10], dyes [11] and explosives [12] among others. Ideal thermal materials are those that do not decompose or melt over a wide temperature range.

Several researchers have used linear and non-linear physical chemistry models to determine transition enthalpies values. In 2001, Chatterjee et al used the linear equation of ClausiusClapeyron to determine the sublimation enthalpy of hydroxyl benzoic acid [13]; while in 2005, Emel'yanenko and Verevkin used a non-linear equation to determine vaporizations enthalpies of amino-toluenes [14]. In this field of study is also typical to calculate the vapor pressure from rate constant values using the Langmuir equation and then to fit the obtained values according to Clausius-Clapeyron equation to find the standard enthalpy values. For example, in 2008, Gupta et al and in 2011, Felix et al calculated vapor pressure from constants rate and determined sublimation enthalpy of Fentanyl and TATP respectively using the Clausius-Clapeyron linear equation [15-16]. On the other hand, others authors have combined spectroscopic and thermal techniques in order to study physical chemistry properties of different materials [1719]. In this work, we are proposing a mathematical analysis of the several thermal model of the pressure and constant evaporation depending on the $\Delta \mathrm{H}$ function. It was modelled the experimental thermal decaying of acetyl salicylic (ASA) and nitro benzoic acid using thermogravimetry analysis in order to corroborate the veracity of the models.

\section{MATHEMATICAL DEDUCTION OF THE THERMAL DECAYING MODEL}

This apart describes the mathematical deduction of the equations that should be used in the fitting of the experimental thermal data (Thermal decaying of vapor pressure or mass lost constant). First step explains the conversion of the Langmuir expression (eq.1) in an equation similar to the ClausiusClapeyron equation in its differential form. In detail, it was resolved for the pressure, applied natural logarithm to both sides, and finally differenced the resultant expression with respect to the reciprocal temperature by obtaining the equation 3. On the other hand, it was replaced, the enthalpy functions in the equations 4-5 and finally, resolving these differential equations were obtained the proposed models to be used in the different fitting of the experimental thermal data. The enthalpy functions were derived from A. Einstein distribution approaching explained below, by integrating the approximated $\Delta \mathrm{Cp}$ Einstein function. All of these equations are summarized in the table 1 .

$$
\begin{aligned}
& \mathrm{k}=\frac{\mathrm{dm}}{\mathrm{dt}}=\mathrm{p \alpha} \sqrt{\frac{\mathrm{M}}{2 \pi \mathrm{RT}}} \\
& \ln \mathrm{p}_{(\mathrm{T})}=\ln \mathrm{k}_{(\mathrm{T})}-\frac{1}{2} \ln \left(\frac{1}{\mathrm{~T}}\right)+\frac{1}{2} \ln \left(\frac{2 \pi \mathrm{R}}{\mathrm{M} \alpha^{2}}\right) \\
& \mathrm{R} \frac{\partial \ln \mathrm{p}_{(\mathrm{T})}}{\partial(1 / \mathrm{T})}=\mathrm{R} \frac{\partial \ln \mathrm{k}_{(\mathrm{T})}}{\partial(1 / \mathrm{T})}-\left(\frac{\mathrm{R}}{2}\right)\left(\frac{1}{\mathrm{~T}}\right)^{-1} \\
& \frac{\partial \ln \mathrm{P}_{(\mathrm{T})}}{\partial(1 / \mathrm{T})}=\frac{-\Delta \mathrm{H}_{\mathrm{T}}}{\mathrm{R}} \\
& \frac{\partial \ln \mathrm{k}(\mathrm{T})}{\partial(1 / \mathrm{T})}=\frac{-\Delta \mathrm{H}_{\mathrm{T}}}{\mathrm{R}}+\frac{1}{2}\left(\frac{1}{\mathrm{~T}}\right)^{-1}
\end{aligned}
$$

$1^{\text {th }}$ LACCEI Annual International Conference: “Engineering Education Facing the Grand Challenges, What Are We Doing?” 
As stated above, the proposed models were obtained using different approaches of the A. Einstein distribution [20], which were considered as follows: at low (below $50^{\circ} \mathrm{K}$ ) and high (above $300^{\circ} \mathrm{K}$ ) temperatures, the heat capacity change at constant pressure $(\mathrm{Cp})$ behaves as a constant, from $30^{\circ} \mathrm{K}$ to $70^{\circ} \mathrm{K}$ behaves as a straight line, and at temperatures between $0^{\circ} \mathrm{K}$ and $70^{\circ} \mathrm{K}$ behaves as a quadratic function; In addition to these approaching, It was also proposed a model using Full Einstein's equation (see Figure 1), by finding the different thermodynamic models of which several of them have been previously used in related studies. An important result, is that the thermodynamical model considering the $\Delta \mathrm{Cp}$ as a constant and the model considering the full Einstein function are significantly similar, which after considering the combined log-exponential term negligible (eq.8), both expressions are identical. The reason why the combined log-exponential term is dismissed, is that at temperatures under Einstein temperature, the term inside the logarithm tends to less than zero numbers, for which the logarithm function is not defined.

$$
\begin{aligned}
& \Delta H=\int\left[R+3 R\left(\frac{a}{T}\right)^{2} \frac{e^{\frac{d}{T}}}{\left(e^{\frac{a}{T}}-1\right)^{2}}\right] d T \\
& \Delta H==\frac{3 a R}{e^{a / T}-1}+b R\left(\frac{1}{T}\right)+c \\
& \ln (p)=\int\left[\frac{3 a}{e^{\frac{a}{T}}-1}+b\left(\frac{1}{T}\right)+\frac{c}{R}\right] d\left(\frac{1}{T}\right) \\
& \ln (p)=a^{s}\left(\frac{1}{T}\right)^{-1}-b^{s} \ln \left(\frac{1}{T}\right)+c^{s}+d \ln \left(1-\frac{\left.e^{\frac{a}{T}}\right)}{}\right. \\
& \ln (p)=a^{s}\left(\frac{1}{T}\right)^{-1}-b^{s} \ln \left(\frac{1}{T}\right)+c^{s}
\end{aligned}
$$

TABLE 1. Condensed thermal function deduced from different consideration of Einstein distribution [20].

\begin{tabular}{|c|}
\hline $\operatorname{Ln}(k)$ function $[10]$ \\
\hline $\ln (k)=-\frac{\mathrm{a}-2}{\mathrm{R}} \ln \left(\frac{1}{\mathrm{~T}}\right)-\frac{\mathrm{b}}{\mathrm{R}}\left(\frac{1}{\mathrm{~T}}\right)+\mathrm{c}$ \\
\hline $\ln (\mathrm{k})=\frac{\mathrm{a}}{2 \mathrm{R}}\left(\frac{1}{\mathrm{~T}}\right)^{-2}-\frac{\mathrm{b}-2}{\mathrm{R}} \ln \left(\frac{1}{\mathrm{~T}}\right)-\frac{\mathrm{c}}{\mathrm{R}}\left(\frac{1}{\mathrm{~T}}\right)$ \\
\hline $\ln (\mathrm{k})=\frac{\mathrm{a}}{6 \mathrm{R}}\left(\frac{1}{\mathrm{~T}}\right)^{-2}-\frac{\mathrm{b}-2}{\mathrm{R}} \ln \left(\frac{1}{\mathrm{~T}}\right)+\frac{\mathrm{c}}{\mathrm{R}}\left(\frac{1}{\mathrm{~T}}\right)$ \\
\hline $\ln (\mathrm{k})=\mathrm{a}^{\mathrm{f}}\left(\frac{1}{\mathrm{~T}}\right)^{-2}-\left(\mathrm{b}^{\prime}-1 / 2 \mathrm{R}\right) \ln \left(\frac{1}{\mathrm{~T}}\right)+\mathrm{c}^{\prime}$ \\
\hline
\end{tabular}

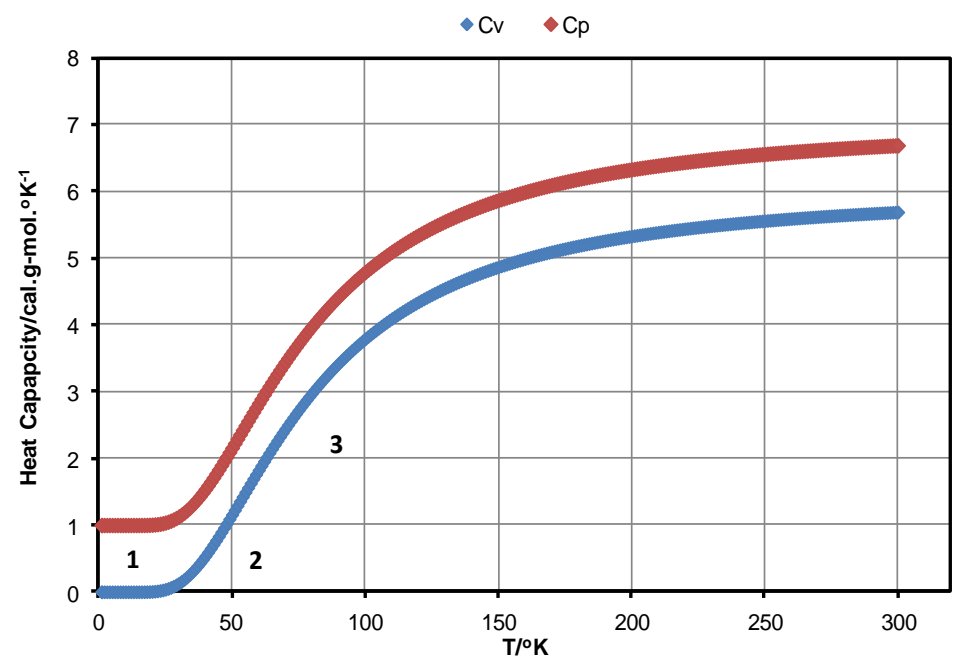

Figure 1. $C_{p}$ and $C_{v}$ Einstein model function of heat capacity, considering $C_{p}$ and $\mathrm{C}_{\mathrm{V}}$ relate by $\mathrm{R}$ constant. The portion 1 is approaching to a constant function. The portion 2 is approaching to a linear function and the portion 3 is approaching to a quadratic function.

\section{COMPARISON OF THE THERMAL MODELS WITH THE EXPERIMENTAL DECAYING}

In this field of study, it is typical to calculate the vapour pressure from rate constant values using the Langmuir equation and then to fit the obtained values according to Clausius-Clapeyron equation to determine standard enthalpy values, assuming that the enthalpy change $(\Delta \mathrm{H})$ is a thermodynamic property independent of the temperature; sometimes this approach works well for some ideal compounds and a narrow ranges of temperature; but others cases not.

The Figures 2-6 show the different thermal profiles depending on the enthalpy function used. In general, in all cases a non-linear trend prevails, when considering wide temperature ranges. As the temperature range decreases, the thermal behavior profiles approximates a straight line. Consequently, each temperature range value will have a different slope in the global curve, which leads to variability in the reported enthalpies. Another important finding, is that the apparently linear trends can also be modeled using mathematical models presented in Table 1 (see Figure 8). On the other hand, in all cases is notable that as the $\Delta \mathrm{Cp}$ value decreases, the thermal profile is getting closer to linear behavior, traditionally used in this field of the thermodynamic chemistry. Although, significant values of $\Delta \mathrm{Cp}$ with an order of magnitude less than the $\Delta \mathrm{H}$ obtained by linear fitting could be fitted using the linear model. 
Fig. 3. Thermal decay of $\ln (\mathrm{p})$ or $\ln (\mathrm{k})$ model using a quadratic enthalpy function in a wide temperature range
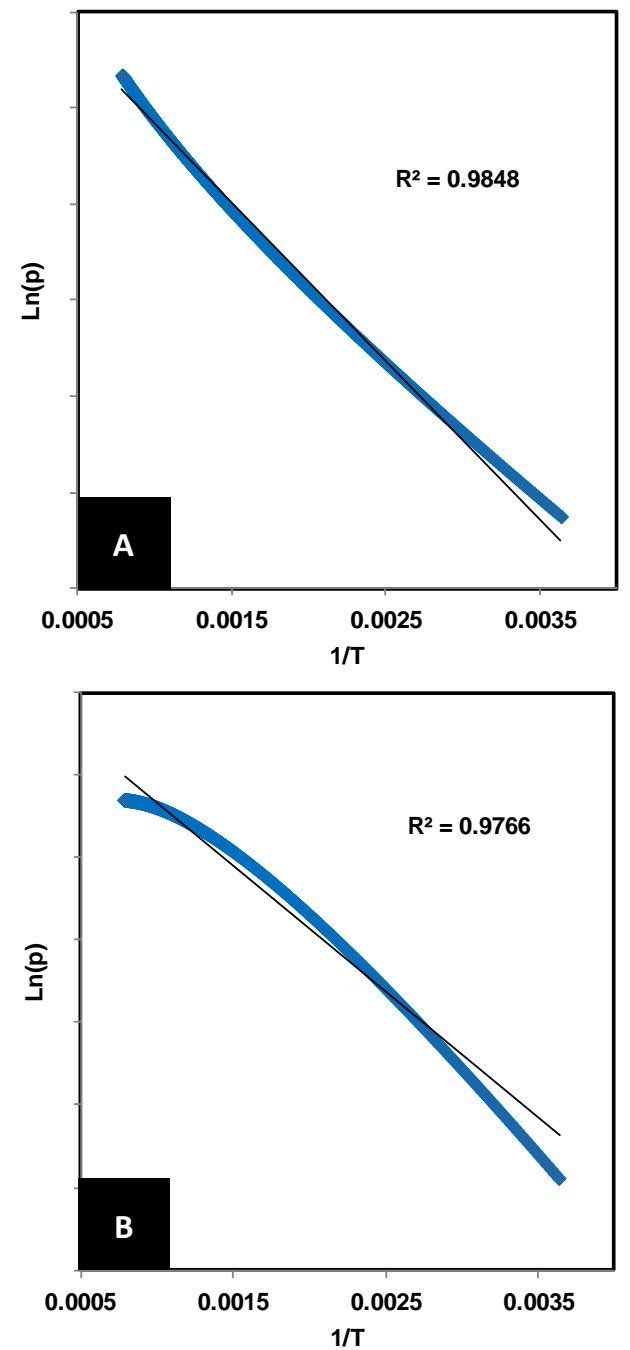

Fig 2. Thermal decay of $\ln (\mathrm{p})$ or $\ln (\mathrm{k})$ model using a linear enthalpy function in a wide temperature range. A. when $\Delta \mathrm{Cp}$ is a positive value, $\mathbf{B}$. When $\Delta \mathrm{Cp}$ is a negative value. It is notable the concavity dependence with the $\Delta \mathrm{Cp}$ sense.

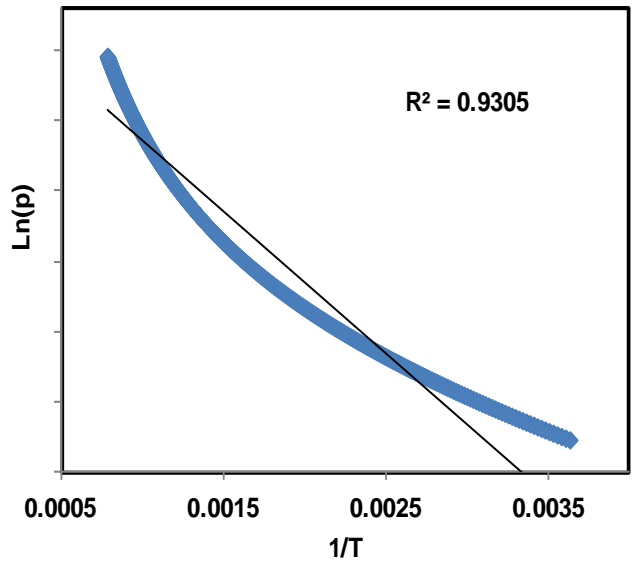

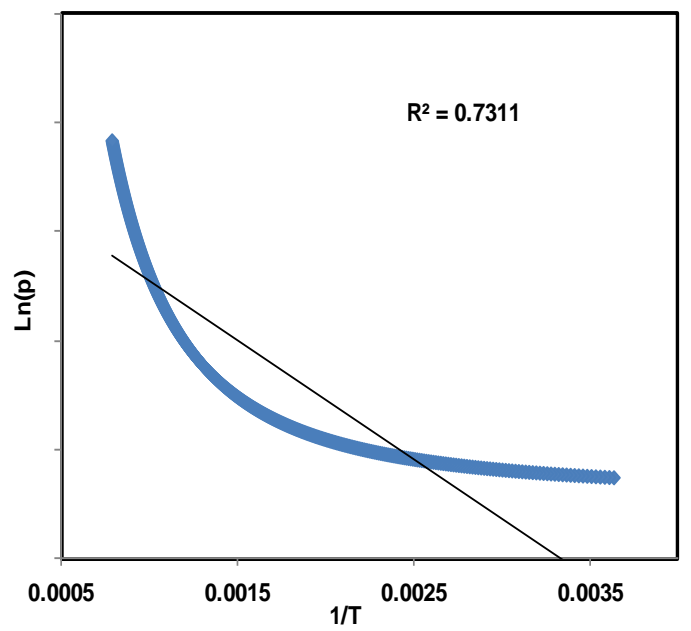

Fig 4. Thermal decay of $\ln (\mathrm{p})$ or $\ln (\mathrm{k})$ model using a cubic enthalpy function in a wide temperature range

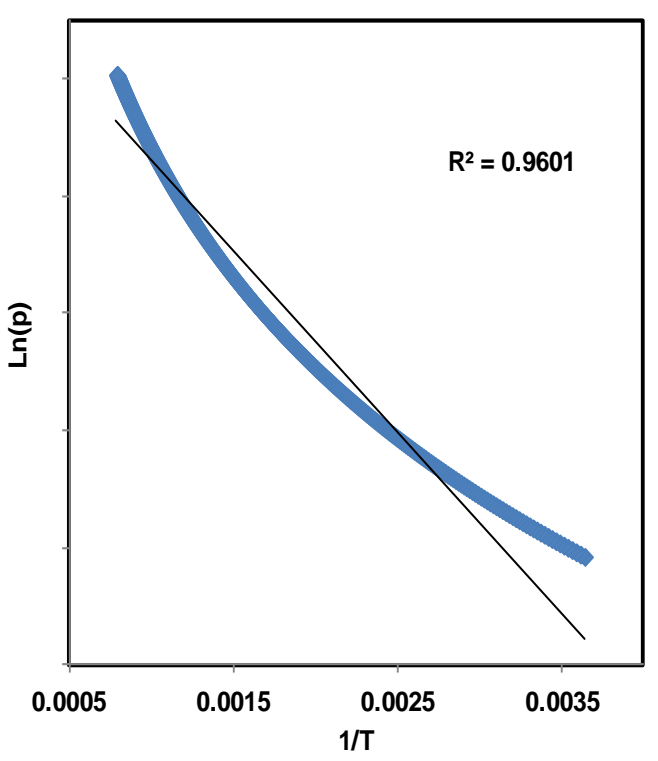

Fig. 5. Thermal decay of $\ln (\mathrm{p})$ or $\ln (\mathrm{k})$ model using an enthalpy function derived from Einstein model in a wide temperature range

Finally, to corroborate the truth of the principles outlined in this manuscript, we found that the equations shown up here perfectly modeled some experimental decays reported in the literature $[8-9,13,21]$ and others tested in our laboratory, as is the case of the compounds nitro benzoic and acetyl salicylic acid, which showed high concordance with respect to model previously postulated reflected in the $\mathrm{R}^{2}$ value of statistical modeling (see Figures 6-7). On the other hand, the models when $\Delta \mathrm{Cp}$ is considered a constant are often published in a linearized form as usual $[8-9,13]$. Another important aspect, is 
that the concavity of global decay depends on the sense of the $\Delta \mathrm{Cp}$ value; when $\Delta \mathrm{Cp}$ is positive, the decaying curve is convex and concave when $\Delta \mathrm{Cp}$ is negative (see Figures $2 \mathrm{~A}$ and $2 \mathrm{~B}$ ). Also, it is notable that as increasing $\Delta \mathrm{Cp}$ value, the concavity of curve is more pronounced, and as $\Delta \mathrm{Cp}$ goes decaying to negligible values, the thermal decay gradually becomes completely linear.

On the other hand, $\Delta \mathrm{H}$ functions helped estimate the magnitude of errors that could be committed in the $\Delta H$ values reported in the literature in a temperature range from $0{ }^{\circ} \mathrm{C}$ to $100^{\circ} \mathrm{C}$, which is the temperature range, where most of the thermodynamic studies are performed. In general, the errors in the enthalpies reported would be approximately one order of magnitude.

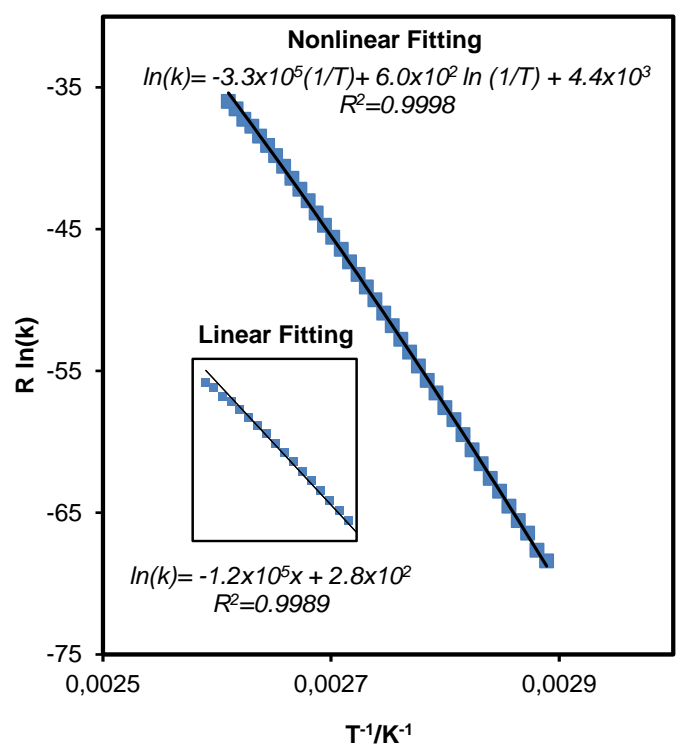

Fig. 6. Experimental decaying of Acetyl salicylic acid. By considering a linear and nonlinear fitting.

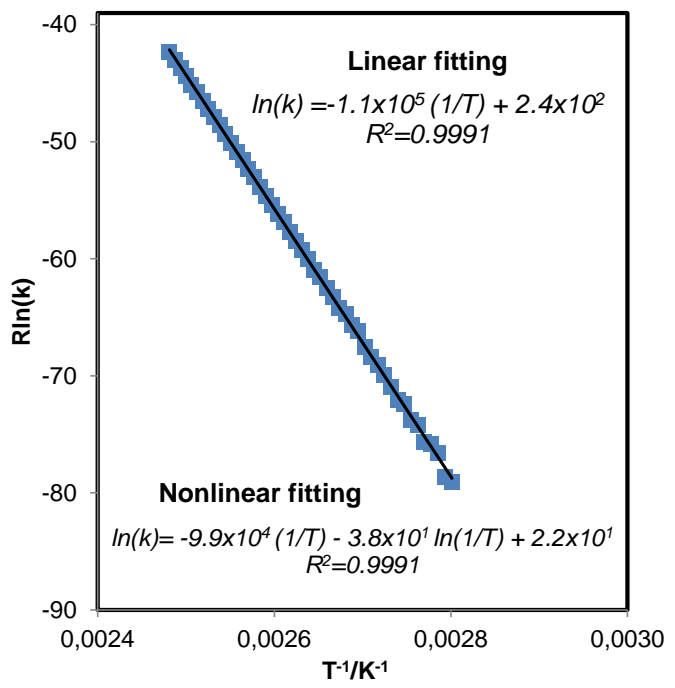

Fig. 7. Experimental thermal fitting of Nitro benzoic acid using a linear and nonlinear model.

\section{CONCLUSION}

In general, all models follow a nonlinear trend at high temperature ranges. The experimental thermal decaying of the compounds nitro benzoic and acetyl salicylic acid showed high concordance with respect to one of the models presented in the Table 1; this approaching is reflected in the $\mathrm{R}^{2}$ value of the statistical modeling, which was closer to one. The theoretical models considering: a $\Delta \mathrm{H}$ linear function and a $\Delta \mathrm{H}$ function derived from Einstein model, presented higher similarity. The thermal experimental decaying of the compounds ASA y nitrobenzoic acid adjusted to the model sketched in the figures 2 and 5. According to reported $\Delta \mathrm{H}$ functions, the errors committed in the reported values would be near to one order a magnitude.

\section{REFERENCES}

[1] Cabalo, J, Sausa, R (2005) Trace detection of explosives with low vapor emissions by laser surface photofragmentation-fragment detection spectroscopy with an improved ionization probe. Appl Opt 44: pp. 10841091

[2] Steinfeld, JI, Wormhoudt, J (1998) Explosives detection: a challenge for physical chemistry. Annu Rev Phys Chem 49: pp. 203-232

[3] Rhykerd CL, Hannum DW, Murray DW, Parmeter JE. Guide for the selection of commercial explosives detection systems for law enforcement applications. Washington, DC: (U.S. Department of Justice) National Institute of Justice; 1999.

[4] The 9/11 commission report: final report of the national commission on terrorist attacks upon the United States. New York: W.W. Norton \& Company; 2004.

[5] Elder, JP (1997) Sublimation measurements of pharmaceutical compounds by isothermal thermogravimetry. J Therm Anal Calorim 49: pp. 897-905

[6] Wright, SF (2004) Determination of the vapor pressure curves of adipic acid and triethanolamine using thermogravimetric analysis. Thermochim Acta 421: pp. 25-30

[7] Xie, M, Ziemba, T, Maurin, M (2003) Sublimation characterization and vapor pressure estimation of an HIV nonnucleoside reverse transcriptase inhibitor using thermogravimetric analysis. AAPS PharmSciTech 4: pp. 99-108

[8] Chatterjee, K, Hazra, A, Dollimore, D, Alexander, KS (2002) Estimating vapor pressure curves by thermogravimetry: a rapid and convenient method for characterization of pharmaceuticals. Eur J Pharm Biopharm 54: pp. 171-180

[9] Chatterjee, K, Dollimore, D, Alexander, KS (2002) Calculation of vapor pressure curves for hydroxy benzoic acid derivatives using thermogravimetry. Thermochim Acta 392: pp. 107-117

[10] Phang, P, Dollimore, D (2001) The calculation of the vapor pressures of antioxidants over a range of temperatures using thermogravimetry. Thermochim Acta 367: pp. 263-268

[11] Price, DM, Hawkins, M (1998) Calorimetry of two disperses dyes using thermogravimetry. Thermochim Acta 315: pp. 19-24

[12] Jones, DEG, Lightfoot, PD, Fouchard, RC, Kwok, QSM (2002) Thermal properties of DMNB, a detection agent for explosives. Thermochim Acta 388: pp. 159-173

[13] Chatterjee, K, Dollimore, D, Alexander, KS (2001) A thermal analysis study of hydroxy benzoic acid derivatives using rising temperature thermogravimetry. J Therm Anal Calorim 63: pp. 629-639

$1^{\text {th }}$ LACCEI Annual International Conference: "Engineering Education Facing the Grand Challenges, What Are We Doing?" 
[14] Emel'yanenko, VN, Verevkin, SP (2005) Enthalpies of formation and substituent effects of ortho, meta, and para aminotoluenes from thermochemical measurements and from Ab initio calculations. J Phys Chem A 109: pp. 3960-3966

[15] Gupta, PK, Ganesan, K, Gutch, PK, Manral, L, Dubey, DK (2008) Vapor pressure and enthalpy of vaporization of fentanyl. J Chem Eng Data 53: pp. 841-845

[16] Abou-Gamra, ZM, Abdel-Messih, MF (2014) Correlation of thermal and spectral properties of chromium (III) picolinate complex and kinetic study of its thermal degradation. J Therm Anal Calorim 117: pp. 993-1000

[17] Grosso, RL, Matos, JR, Muccillo, ENS (2014) Thermal and spectroscopic characterization of nanostructured zirconia-scandiadysprosia. J Therm Anal Calorim 117: pp. 567-572

[18] Jethva, HO, Vyas, PM, Tank, KP, Joshi, MJ (2014) FTIR and thermal studies of gel-grown, lead-cadmium-mixed levo tartrate crystals. J Therm Anal Calorim 117: pp. 589-594

[19] Elementary solid state physics. Massachusetts: Addison-Wesley Publishing Company; 1975. pp. 79-80.

[20] Espinosa-Fuentes, EA, Castro-Suarez, JR, Meza-Payares, DD, PachecoLondono, LC, Hernández-Rivera, SP (2015) J Therm Anal Calorim 119: pp.681-688

13 ${ }^{\text {th }}$ LACCEI Annual International Conference: "Engineering Education Facing the Grand Challenges, What Are We Doing?" July 29-31, 2015, Santo Domingo, Dominican Republic 